\title{
The Khavinson-Shapiro conjecture for domains with a boundary consisting of algebraic hypersurfaces
}

\author{
Hermann Render \\ School of Mathematics and Statistics, University College Dublin, \\ Belfield, Dublin 4, Ireland.
}

\begin{abstract}
The Khavinson-Shapiro conjecture states that ellipsoids are the only bounded domains in euclidean space satisfying the following property (KS): the solution of the Dirichlet problem for polynomial data is polynomial. In this paper we show that a domain does not have property $(\mathrm{KS})$ provided the boundary contains at least three differrent irreducible algebraic hypersurfaces for which two of them have a common point.
\end{abstract}

\section{Introduction}

A complex-valued function $h$ defined on an open set $\Omega$ in $\mathbb{R}^{d}$ is called $h a r$ monic if $h$ is differentiable of order 2 and satisfies the equation $\Delta h(x)=0$ for all $x \in \Omega$ where

$$
\Delta=\frac{\partial^{2}}{\partial x_{1}^{2}}+\cdots+\frac{\partial^{2}}{\partial x_{d}^{2}}
$$

is the Laplacian. The Khavinson-Shapiro conjecture in [18] states that ellipsoids are the only bounded domains $\Omega$ in $\mathbb{R}^{d}$ with the following property:

(KS) For any polynomial $p$ there exists a harmonic polynomial $h$ such that $h(\xi)=p(\xi)$ for all $\xi \in \partial \Omega$.

Obviously a domain $\Omega$ has property (KS) if and only if the Dirichlet problem for polynomial data (restricted to the boundary) has polynomial solutions; for more details on the Dirichlet problem we refer the reader to [5] and [14]. The Khavinson-Shapiro conjecture has been confirmed for large classes of domains but it is still unproven in its full generality, and we refer the interested reader to [15, [23, [20, [16], 21], [17] and for further ramifications in 19 ] originating from the work in 22 .

\footnotetext{
${ }^{1} 2010$ Mathematics Subject Classification 31B05; 35J05. Keywords and phrases: Dirichlet problem, harmonic extension, Khavinson-Shapiro conjecture.
} 
For certain classes of domains $\Omega$ one can prove that the solution of the Dirichlet problem for the polynomial

$$
|x|^{2}=x_{1}^{2}+\cdots+x_{d}^{2}
$$

develops a singularity in $\mathbb{R}^{d}$, in particular it is not a polynomial and $\Omega$ does not have property (KS). Instructive is a beautiful result of P. Ebenfeld in [1] for the "TV-screen" domain

$$
\Omega=\left\{(x, y) \in \mathbb{R}^{2}: x^{4}+y^{4}<1\right\}
$$

where the solution of the Dirichlet problem $u(x, y)$ for the data function $x^{2}+y^{2}$ can be extended to a real-analytic function on $\mathbb{R}^{2} \backslash D$ where $D$ is a countable discrete set. Further illuminating examples of domains satisfying the Khavinson-Shapiro conjecture have been provided by E. Lundberg in [20] using lightning bolts as a mayor technique.

In this paper we want to discuss the Khavinson-Shapiro conjecture for domains which consists of finitely many algebraic hypersurfaces. The simplest example of a such domain is a polygonal domain in the plane. E.A. Volkov proved in [27] the following result: An equilateral triangle is the unique polygon $D$ in the plane $x y$ on which the solution of the boundary problem

$$
\Delta u=1 \text { on } D \text { and } u=0 \text { on } \partial D
$$

is an algebraic polynomial, and moreover, the degree of this polynomial is equal to 3. Note that for a solution $u$ of (11) the function $v(x, y)=$ $x^{2}+y^{2}-4 u(x, y)$ is harmonic and equal to $x^{2}+y^{2}$ on $\partial D$. It follows that the only polygonal domain for which the polynomial $x^{2}+y^{2}$ has a polynomial solution for the Dirichlet problem is an equilateral triangle. We shall prove in this paper that no polygonal domain has property (KS). Thus the equilateral triangle is an example of a domain where the polynomial $x^{2}+y^{2}$ has a polynomial solution for the Dirichlet problem but property (KS) is not satisfied (for further examples see [15] or [21]).

Let us denote the set of all polynomials in $d$ variables with real coefficients by $\mathbb{R}[x]$. Assume that $\psi_{1}, \ldots, \psi_{N} \in \mathbb{R}[x]$. We say that the boundary $\partial \Omega$ of a domain $\Omega$ in $\mathbb{R}^{d}$ contains $N$ different irreducible algebraic hypersurfaces defined by $\psi_{1}, \ldots, \psi_{N}$ if (i) each polynomial $\psi_{k}, k=1, \ldots N$ is irreducible, (ii) $\psi_{k} \neq \lambda \psi_{l}$ for all $k \neq l, k, l=1, \ldots, N$ and for all real numbers $\lambda$, and (iii) for each $k=1, \ldots, N$ there exists an open set $U_{k}$ such that

$$
\left\{x \in \mathbb{R}^{d}: \psi_{k}(x)=0\right\} \cap U_{k} \subset \partial \Omega
$$

and $\psi_{k}$ changes its sign on $U_{k}$, i.e. there exist $x, y \in U_{k}$ such that $\psi_{k}(x)<$ $0<\psi_{k}(y)$. Obviously a polygonal domain in the plane with $N$ edges satisfies this condition where the polynomials $\psi_{k}, k=1, \ldots, N$ are linear polynomials.

Elementary results in Algebraic Geometry (cf. the proof of Theorem 27 in [23]) lead to the following observation: 
Theorem 1 Assume that $\partial \Omega$ contains $N$ different irreducible algebraic hypersurfaces defined by the polynomials $\psi_{1}, \ldots, \psi_{N}$. If $\Omega$ has property (KS) then the Fischer operato $2^{2} F_{\psi}: \mathbb{R}[x] \rightarrow \mathbb{R}[x]$ defined by

$$
F_{\psi}(q):=\Delta(\psi q) \text { for } q \in \mathbb{R}[x]
$$

is surjective where $\psi=\psi_{1} \cdot \ldots \cdot \psi_{N}$.

The main result of this paper is the following:

Theorem 2 Suppose that the polynomial $\psi$ has at least three non-constant polynomial factors and two of the factors have a common zero $x_{0}$. Then $F_{\psi}$ is not surjective.

As an application we see that no bounded domain $\Omega$ in $\mathbb{R}^{2}$ with polygonal boundary has property (KS): there are at least 3 edges defined by linear polynomials $\psi_{1}, \psi_{2}, \psi_{3}$. Since $\Omega$ is bounded at least two of them have a common zero and the result follows. In particular, the equilateral triangle does not satisfy property (KS).

Theorem 1 shows that (KS) is related to the following purely algebraic question: for which polynomials $\psi \in \mathbb{R}[x]$ is the Fischer operator $F_{\psi}$ : $\mathbb{R}[x] \rightarrow \mathbb{R}[x]$ defined by

$$
F_{\psi}(q):=\Delta(\psi q) \text { for } q \in \mathbb{R}[x]
$$

surjective? In [10] M. Chamberland and D. Siegel formulated the following conjecture:

(CS) The surjectivity of the Fischer operator $F_{\psi}: \mathbb{R}[x] \rightarrow \mathbb{R}[x]$ implies that the degree of $\psi$ is $\leq 2$.

In the last section we will summarize a recent result of the author showing that (CS) implies the Khavinson-Shapiro conjecture for so-called admissible domains. We refer the interested reader to [21] for more results on the conjecture (CS) and related statements.

\section{Surjectivity of Fischer operators.}

The degree of a polynomial $\psi$ is denoted by $\operatorname{deg} \psi$. Recall that the multiplicity $N$ of a zero $x_{0}$ of a polynomial $\psi(x)$ is the largest natural number $N$ such that

$\frac{\partial^{\alpha}}{\partial x^{\alpha}} \psi\left(x_{0}\right)=0$ for all $\alpha=\left(\alpha_{1}, \ldots, \alpha_{d}\right) \in \mathbb{N}_{0}^{d}$ with $|\alpha|=\alpha_{1}+\ldots+\alpha_{d} \leq N-1$.

\footnotetext{
${ }^{2}$ More generaly one can define a Fischer operator by $q \longmapsto P(D)(\psi q)$ where $P(D)$ is a linear partial differential operator with constant real coefficients. Fischer's Theorem in 13 states that the Fischer operator is a bijection whenever $\psi(x)$ is a homogeneous polynomial equal to the polynomial $P(x)$.
} 
A polynomial $f_{j}$ is called homogeneous of degree $j$ if $f_{j}(r x)=r^{j} f_{j}(x)$ for all $r>0$ and for all $x \in \mathbb{R}^{d}$. A polynomial $f$ of degree $m$ can be expanded into a finite sum of homogeneous polynomials $f_{j}$ of degree $j$ such that

$$
f=f_{t}+\ldots+f_{m} \text { and } t \leq m
$$

where $f_{m} \neq 0$ is called the principal part or leading part, and $f_{t} \neq 0$ is called the initial part of the polynomial $f$.

The following definition arises in the investigation of stationary sets for the wave and heat equation, see [1, 2], and the injectivity of the spherical Radon transform, see [3], and the investigation of level curves of harmonic functions, see [12, p. 425].

Definition 3 A polynomial $f$ is called a harmonic divisor if there exists a non-zero polynomial $q$ such that $f q$ is harmonic.

Thus a polynomial is harmonic divisor if and only if is a factor of a harmonic polynomial, in particular every harmonic polynomial is a harmonic divisor (by taking $q$ as the constant function).

By Theorem 2.2.3 in [5] any harmonic divisor takes both positive and negative values, thus a harmonic divisor does not have any non-negative factor, a result which is due to M. Brelot and G. Choquet in [9].

The following result is well known:

Proposition 4 Let $f$ be a polynomial with homogeneous expansion $f=$ $f_{t}+\ldots+f_{m}$, where $f_{t} \neq 0$ and $f_{m} \neq 0$. If $f$ is a harmonic divisor then $f_{t}$ and $f_{m}$ are harmonic divisors.

The next two results provide some simple necessary conditions of the surjectivity of the Fischer operator:

Proposition 5 Let $\psi$ be a polynomial of degree $\geq 3$ with homogeneous expansion

$$
\psi=\psi_{t}+\ldots+\psi_{m},
$$

where $\psi_{t} \neq 0$ and $\psi_{m} \neq 0$. If the constant function is in the image of the Fischer operator $F_{\psi}$ then $\psi_{m}$ is a harmonic divisor.

Proof. For the constant function 1 there exists a polynomial $q$ such that $1=\Delta(\psi q)$. Let us write $q=q_{T}+\ldots .+q_{M}$ with $q_{T} \neq 0$ and $q_{M} \neq 0$. Then the polynomial $\psi q$ has the homogeneous expansion

$$
\psi q=\psi_{t} q_{T}+\left(\psi_{t+1} q_{T}+\psi_{t} q_{T+1}\right)+\cdots+\psi_{m} q_{M} .
$$

By assumption $1=\Delta(\psi q)$, i.e. the homogeneous expansion of the polynomial $\Delta(\psi q)$ consists just of the constant function 1 . On the other hand, $\psi_{m} q_{M}$ has degree at least 3 , so $\Delta\left(\psi_{m} q_{M}\right)$ has degree 1, and therefore $\Delta\left(\psi_{m} q_{M}\right)=$ 0 . 
Proposition 6 Let $\psi$ be a polynomial. If the constant function is in the image of the Fischer operator $F_{\psi}$ then any zero $x \in \mathbb{R}^{d}$ of $\psi$ has multiplicity $\leq 2$.

Proof. Let $q$ be a polynomial such that $1=F_{\psi}(q)=\Delta(\psi q)$. If the multiplicity of $\psi$ at $x_{0}$ is larger than 2 then all derivatives of $\psi$ of order $\leq 2$ at $x_{0}$ are zero and therefore

$$
\frac{\partial^{2}}{\partial x_{j}^{2}}(\psi q)\left(x_{0}\right)=0
$$

for $j=1, \ldots, d$. Thus $\Delta(\psi q)\left(x_{0}\right)=0$, a contradiction.

From [21] we cite the following result which is known as the Fischer decomposition of a polynomial.

Proposition 7 Suppose $\psi$ is a polynomial. Then the operator $F_{\psi}(q):=$ $\Delta(\psi q)$ is surjective if and only if every polynomial $f$ can be decomposed as $f=\psi q_{f}+h_{f}$, where $q_{f}$ is a polynomial and $h_{f}$ is harmonic polynomial

In the following we always assume that the polynomial $\psi$ in the definition of the Fischer operator $F_{\psi}$ has at least two factors:

Proposition 8 Suppose that the polynomial $\psi$ has at least two non-constant polynomial factors, say $\psi=\psi^{(1)} \psi^{(2)}$. If $F_{\psi}$ is surjective then $\psi^{(1)}$ and $\psi^{(2)}$ are harmonic divisors.

Proof. We can write $\psi^{(1)}=\psi^{(1)} \psi^{(2)} q+h$ for some polynomial $q$ and a harmonic polynomial $q$. Then $h \neq 0$ since otherwise $\psi^{(1)}=\psi^{(1)} \psi^{(2)} q$ which is impossible since $\psi^{(2)}$ has degree $\geq 1$. It follows that for $g:=1-\psi^{(2)} q$

$$
\psi^{(1)} g=h \neq 0 .
$$

Then $g \neq 0$ and $\psi^{(1)}$ is a harmonic divisor. The case of $\psi^{(2)}$ follows by symmetry of the problem.

Lemma 9 Let $x_{0} \in \mathbb{R}^{d}$. Then the Fischer operator $F_{\psi}: \mathbb{R}[x] \rightarrow \mathbb{R}[x]$ is surjective if and only if $F_{\varphi}: \mathbb{R}[x] \rightarrow \mathbb{R}[x]$ is surjective for the polynomial $\varphi(x)=\psi\left(x-x_{0}\right)$.

Proof. Let $f(x)$ be polynomial and define $g(x)=f\left(x+x_{0}\right)$. If $F_{\psi}$ is surjective then there exist a polynomial $q$ and a harmonic polynomial $h$ such that $g(x)=\psi(x) q(x)+h(x)$ for all $x \in \mathbb{R}^{d}$. Replace $x$ by $x-x_{0}$, then

$$
f(x)=\psi\left(x-x_{0}\right) q\left(x-x_{0}\right)+h\left(x-x_{0}\right) .
$$

Clearly $x \longmapsto h\left(x-x_{0}\right)$ is harmonic. It follows that $F_{\varphi}$ is surjective. The converse follows similarly.

If $F_{\psi}$ is surjective and if $\psi$ has a zero of multiplicity 2 at some $x_{0}$ then Lemma 9 shows that we may assume that $x_{0}=0$. In this case the following observation is crucial: 
Theorem 10 Suppose that $\psi$ has a zero of multiplicity 2 at 0 and let

$$
\psi(x)=\psi_{2}(x)+\ldots+\psi_{t}(x)
$$

be the expansion into a sum of homogeneous polynomials with $\psi_{2} \neq 0$ and $\psi_{t} \neq 0$. If the Fischer operator $F_{\psi}: \mathbb{R}[x] \rightarrow \mathbb{R}[x]$ is surjective then the Fischer operator $F_{\psi_{2}}: \mathbb{R}[x] \rightarrow \mathbb{R}[x]$ with respect to the polynomial $\psi_{2}$ of degree 2 is bijective. Moreover the Fischer operator $F_{\psi}: \mathbb{R}[x] \rightarrow \mathbb{R}[x]$ is bijective.

Proof. Let $\mathbb{P} \leq N$ be the space of all polynomials of degree $\leq N$. We claim by induction over $N$ that the operator $F_{\psi_{2}}: \mathbb{P} \leq N \rightarrow \mathbb{P} \leq N$ is a bijection. For $N=0$, the space $\mathbb{P} \leq 0$ consists of multiples of the constant polynomials. By surjectivity of $F_{\psi}$ there exists a polynomial $q$ such that $1=\Delta(\psi q)$. Let us write $q=q_{0}+\ldots .+q_{s}$. Then

$$
\psi q=\psi_{2} q_{0}+\text { higher order terms. }
$$

Since $1=\Delta(\psi q)$ we infer that $1=\Delta\left(\psi_{2} q_{0}\right)=F_{\psi_{2}}\left(q_{0}\right)$. Thus $F_{\psi_{2}}: \mathbb{P} \leq 0 \rightarrow$ $\mathbb{P} \leq 0$ is surjective and therefore bijective. Assume that the statement is true for $N-1$ and we want to prove it for $N$. By induction hypothesis and the linearity of $F_{\psi_{2}}: \mathbb{P}^{\leq N} \rightarrow \mathbb{P} \leq N$ it suffices to show that for each nonzero homogeneous polynomial $f_{N}$ of degree $N$ there exists $q \in \mathbb{P} \leq N$ with $f_{N}=F_{\psi_{2}} q$. Since $F_{\psi}$ is surjective there exists a polynomial $q$ such that $F_{\psi}(q)=f_{N}$. We write $q$ has a sum of homogeneous polynomials $q_{j}$, say

$$
q=q_{m}+\ldots .+q_{M}
$$

where $q_{m} \neq 0$ and $q_{M} \neq 0$. Then

$$
\psi q=\psi_{2} q_{m}+G_{m+3}+\ldots .+G_{t+N}
$$

where $G_{m+j}$ are homogeneous polynomials of degree $m+j$ for $j=3, \ldots, n$. If $m<N$ then $\Delta\left(\psi_{2} q_{m}\right)=0$ since $\Delta(\psi q)=f_{N}$. By induction hypothesis $F_{\psi_{2}}: \mathbb{P} \leq N-1 \rightarrow \mathbb{P} \leq N-1$ is bijective, so we conclude that $q_{m}=0$, a contradiction. Hence $m \geq N$. If $m>N$ then all homogeneous summands of $\psi q$ have degree at least $m+2>N$, and from $\Delta(\psi q)=f_{N}$ we obtain that $f_{N}=0$, a contradiction. It follows that $m=N$ and $f_{N}=\Delta\left(\psi_{2} q_{N}\right)$. Thus $F_{\psi_{2}}$ is bijective.

Suppose that $F_{\psi}$ is not injective. Then there exists a polynomial $q$ such that $\Delta(\psi q)=0$, so $\Delta\left(\psi_{2} q_{m}\right)=0$ for some homogeneous polynomial $q_{m} \neq 0$. Since $F_{\psi_{2}}$ is injective we infer that $q_{m}=0$, a contradiction.

Theorem 11 Suppose that the polynomial $\psi$ of degree $\geq 3$ has two nonconstant polynomial factors, say $\psi=\psi^{(1)} \psi^{(2)}$. If there exists a zero $x_{0}$ of $\psi^{(1)}$ of multiplicity $\geq 2$ then $F_{\psi}$ is not surjective. 
Proof. Suppose that $F_{\psi}$ is surjective. Proposition [6 show that $x_{0}$ is a zero of $\psi$ of multiplicity $\leq 2$. Since $\psi^{(1)}$ has a zero of multiplicity 2 at $x_{0}$ it follows that $\psi^{(2)}$ has not a zero at $x_{0}$. We may assume that $\psi^{(2)}(0)=$ 1. By Proposition $8 \psi^{(1)}$ is a harmonic divisor, and by Proposition 5 the initial part $\psi_{2}^{(2)}$ of the homogeneous expansion of $\psi_{2}^{(1)}$ is a harmonic divisor. Since $\psi^{(2)}(0)=1$ it follows that $\psi_{2}^{(1)}$ is initial part of the homogeneous expansion of $\psi$ which was denoted by $\psi_{2}$. Thus $\psi_{2}$ is a harmonic divisor which contradicts to the fact $F_{\psi_{2}}$ is bijective.

Finally we present the proof of the main result of the paper:

Theorem 12 Suppose that the polynomial $\psi$ has at least three non-constant polynomial factors and two of the factors have a common zero $x_{0}$. Then $F_{\psi}$ is not surjective.

Proof. Let $\psi=\varphi_{1} \varphi_{2} \varphi_{3}$ for some non-constant polynomial $\varphi_{j}$ for $j=1,2,3$. Suppose that $x_{0}$ is a common zero of two factors, say $\varphi_{1}\left(x_{0}\right)=0$ and $\varphi_{2}\left(x_{0}\right)=0$. Then the product $\varphi_{1} \varphi_{2}$ has a zero at $x_{0}$ of multiplicity $\geq 2$. By Theorem 11 the Fischer operator is not surjective.

\section{On the conjecture (CS)}

The zero-set of a polynomial $f$ will be denoted by $Z(f)=\left\{x \in \mathbb{R}^{d}: f(x)=0\right\}$. We say that $Z$ is an admissible common zero set if there exists non-constant irreducible polynomials $f, g \in \mathbb{R}[x]$ such that (i) $f \neq \lambda g$ for all $\lambda \in \mathbb{R}$ and (ii) $Z=Z(f) \cap Z(g)$. Now we define the class of domains for which we can characterize the property (KS):

Definition 13 An open $\Omega$ in $\mathbb{R}^{d}$ is admissible if for any $x \in \partial \Omega$, any open neighborhood $V$ of $x$ and for any finite family of admissible common zero sets $Z_{1}, \ldots, Z_{r}$ the set

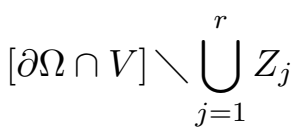

is non-empty.

For dimension $d=2$ it is easy to see that an open set $\Omega$ is admissible if and only if each boundary point $x \in \partial \Omega$ is not isolated in $\partial \Omega$ since an admissible common zero set is finite. For arbitrary dimension $d$ it is intuitively clear that an admissible common zero set has dimension $d-2$. Thus if the boundary $\partial \Omega$ has dimension $d-1$ we expect that the domain $\Omega$ is admissible. However, it seems to be difficult to formulate this as a precise topological condition.

In [24] we presented the following result: 
Theorem 14 Let $\Omega$ be an open admissible subset of $\mathbb{R}^{d}$. Then property (KS) holds for $\Omega$ if and only if there exists a non-constant polynomial $\psi \in \mathbb{R}[x]$ such that (i) $\partial \Omega \subset \psi^{-1}\{0\}$ and (ii) the Fischer operator $F_{\psi}: \mathbb{R}[x] \rightarrow \mathbb{R}[x]$ defined by

$$
F_{\psi}(q):=\Delta(\psi q) \text { for } q \in \mathbb{R}[x]
$$

is surjective.

It follows that for admissible domains the conjecture (CS) implies the Khavinson-Shapiro conjecture.

\section{References}

[1] M.L. Agranovsky, Y. Krasnov, Quadratic Divisors of Harmonic polynomials in $\mathbb{R}^{n}$, J. D'Anal. Math. 82 (2000), 379-395.

[2] M.L. Agranovsky, E.T. Quinto, Geometry of stationary sets for the wave equation in $\mathbb{R}^{n}$. The case of finitely supported initial data, Duke Math. J. 107 (2001), 57-84.

[3] M.L. Agranovsky, V.V. Volchkov, L.A. Zalcman, Conical Uniqueness sets for the spherical Radon Transform, Bull. London Math. Soc. 31 (1999), 231-236.

[4] D. H. Armitage, The Dirichlet problem when the boundary function is entire, J. Math. Anal. Appl. 291 (2004), 565-577.

[5] D. H. Armitage, S. J. Gardiner, Classical Potential Theory, Springer, London 2001.

[6] S. Axler, P. Bourdon, W. Ramey, Harmonic Function Theory, 2nd Edition, Springer, 2001.

[7] S. Axler, P. Gorkin, K. Voss, The Dirichlet problem on quadratic surfaces, Math. Comp. 73 (2003), 637-651.

[8] J. A. Baker, The Dirichlet problem for ellipsoids, Amer. Math. Monthly, 106 (1999), 829-834.

[9] M. Brelot, G. Choquet, Polynomes harmoniques et polyharmoniques, Second colloque sur les 'equations aux d'eriv'ees partielles, Bruxelles, (1954) 45-66.

[10] M. Chamberland, D. Siegel, Polynomial solutions to Dirichlet problems, Proc. Amer. Math. Soc. 129 (2001), 211-217.

[11] P. Ebenfelt, Singularities encountered by the analytic continuation of solutions to Dirichlet's problem, Complex Variables 20 (1992), 75-91. 
[12] L. Flatto, D.J. Newman, H.S. Shapiro, The level curves of harmonic functions, Trans. Amer. Math. Soc. 123 (1966), 425-436.

[13] E. Fischer, Über die Differentiationsprozesse der Algebra, J. für Math. (Crelle Journal) 148 (1917), 1-78.

[14] S.J. Gardiner, The Dirichlet problem with non-compact boundary, Math. Z. 213 (1993), 163-170.

[15] L. Hansen, H.S. Shapiro, Functional Equations and Harmonic Extensions, Complex Variables, 24 (1994), 121-129.

[16] D. Khavinson, E. Lundberg, The search for singularities of solutions to the Dirichlet problem: recent developments, Hilbert spaces of analytic functions, 121-132, CRM Proc. Lecture Notes, 51, Amer. Math. Soc., Providence, RI, 2010.

[17] D. Khavinson, E. Lundberg, A tale of ellipsoids in Potential Theory, Notices Amer. Math. Soc. 61 (2014), 148-156.

[18] D. Khavinson, H. S. Shapiro, Dirichlet's Problem when the data is an entire function, Bull. London Math. Soc. 24 (1992), 456-468.

[19] D. Khavinson, N. Stylianopoulos, Recurrence relations for orthogonal polynomials and algebraicity of solutions of the Dirichlet problem, to appear in "Around the Research of Vladimir Maz'ya II, Partial Differential Equations", 219-228, Springer, 2010.

[20] E. Lundberg, Dirichlet's problem and complex lightning bolts, Comp. Meth. Function Theory 9 (2009), 111-125.

[21] E. Lundberg, H. Render, The Khavinson-Shapiro conjecture and polynomial decompositions, J. Math. Anal. Appl. (2010), 506-513.

[22] M. Putinar, N. Stylianopoulos, Finite-term relations for planar orthogonal polynomials, Complex Anal. Oper. Theory 1 (2007), 447-456.

[23] H. Render, Real Bargmann spaces, Fischer decompositions and sets of uniqueness for polyharmonic functions, Duke Math. J. 142 (2008), 313352.

[24] H. Render, A characterization of the Khavinson-Shapiro conjecture via Fischer operators, submitted.

[25] H.S. Shapiro, An algebraic theorem of E. Fischer and the Holomorphic Goursat Problem, Bull. London Math. Soc. 21 (1989), 513-537.

[26] E.A. Volkov, On differential properties of solutions to boundary value problems for the Laplace equation on polygons, Trudy Mat. Inst. Steklov [Proc. Steklov Inst. Math.], 77 (1965), 113-142. 
[27] E.A. Volkov, On a property of solutions to the Poisson equation on polygons, Mathematical Notes 66 (1999), 139-141 (translated from Matematicheskie Zametki 66 (1999), 178-180). 